\title{
GLOBAL CHARACTERIZATION OF ROSSBY WAVES AT SEVERAL SPECTRAL BANDS
}

Paulo S. Polito ${ }^{1}$ and W. Timothy Liu

Earth Observation Department

INPE - National Institute for Space Research, Brazil
2 Jet Propulsion Laboratory

California Institute of Technology
INTRODUCTION

Rossby waves are the ocean's response to large scale perturbations, based on conservation of potential vorticity. Typically these waves are $\sim 1,000-10,000 \mathrm{~km}$ long, have a period of months to years

As a first approximation the ocean behaves as a two-layer system with the vertical displacement of the interface induced by Rossby waves. These vertical displacements are $\sim 10-100 \mathrm{~m}$.

These long, baroclinic waves are non-dispersive and transport energy westward to help maintain the mid-latitude gyres and to intensify the western boundary currents. The energy and the phase propa-

The TOPEX/Poseidon altimeter (T/P) provided for the first time a long global time series of the sea surface height anomaly $(\eta)$. Re [Zang and Wunch(1999)] (ZW) raised an interesting debate over the validity of the standard linear theory to estimate the Rossby wave phase speed.

In this study a series of finite impulse response (FIR) filters are used to separate the T/P $\eta$ into several dynamical components. The

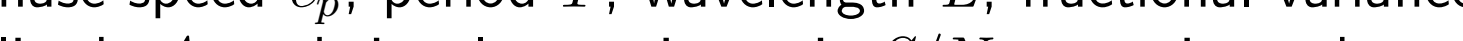

The same technique has been successfully applied to compare heat storage from T/P and in situ data in [Polito et al.(2000)] since Radon transform technique [Polito and Cornillon(1997)] was used

\section{METHODS}

The bin-averaged $\eta$ data from the WOCE dataset (JPL/PODAAC) has the 8-year mean (93-00) removed and are for the Pacific, Atlantic, and Indian basins are converted to zonalTheral diarams of $\eta(x, t)$, one per latitude.

The $\eta_{o}$ is decomposed through FIR filters into:

- in to $\eta_{3}$ are long firt 6 mode Rosshy waves with apporinate periods of 24, 12, 6, and 3 months.

$\eta_{1}$ has a period of 1.5 months and is dominated by tropical instability waves (TIWs).

$\eta_{K}$ is present only in the equatorial region as a fast eastward propagating semiannual signal identified as Kelvin waves

be identes meso-scale eddies

$\eta_{r}$ is dominated by small scale, high frequency residual.
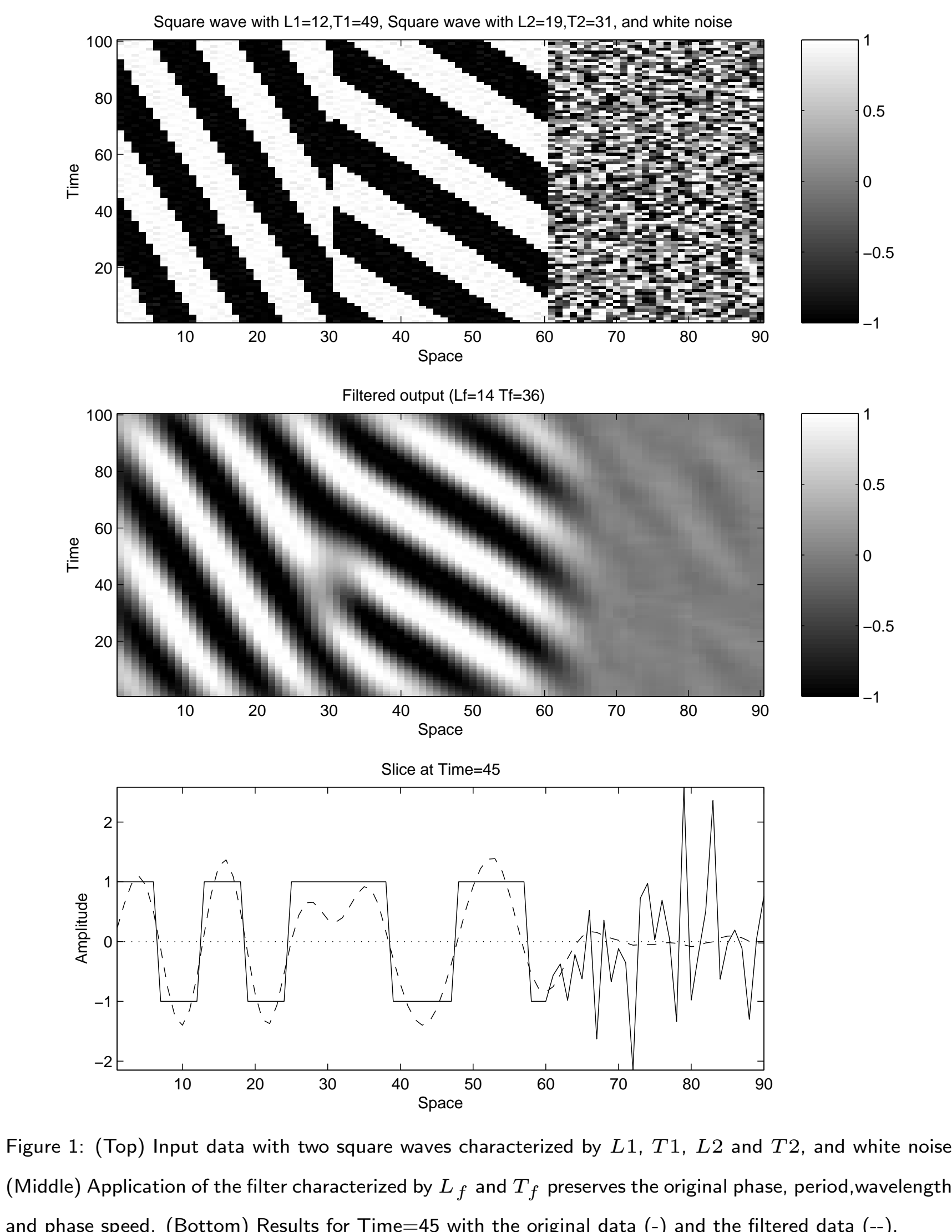

Figure 1 shows an example of the filter performance. Two squarewaves and a random noise field form a single matrix. This matrix is data. The filter period, wavelength, and phase speed are slightly difthat:

- filtering does not change the $C_{j} T, L_{1}$ or $A$ of the original signal.

- even when the filter does not exactly match the wave charbandwidth).

- the filter does not create signals from noise,

- no particular wave form is assumed or enforced.

\section{RESULTS}

\section{A series of FIR filters is applied to $\eta_{0}$ to obtai}

作 Figure 2 shows the filtered fields and the average $c_{p}$ at $28.5^{\circ} \mathrm{N}$ in as are aligned, in average, with the propagation patterns.

In Figure 3 the wave regimes that characterize the equatorial $\mathrm{Pa}$ cific are shown. The basin-scale component $\eta_{t}$ is dominated by ENSO which also modulates the $\eta_{1}$ signal, associated with tropical instability From the filtered components shown in Figures 2 and 3 the wave parameters for each data block measuring approximately $T$ by $L$ are estimated.

Figure 4 shows the rms amplitude of the filtered $\eta$ components intensity of these fields.

The wave par
ures 5,6 , and 7 .

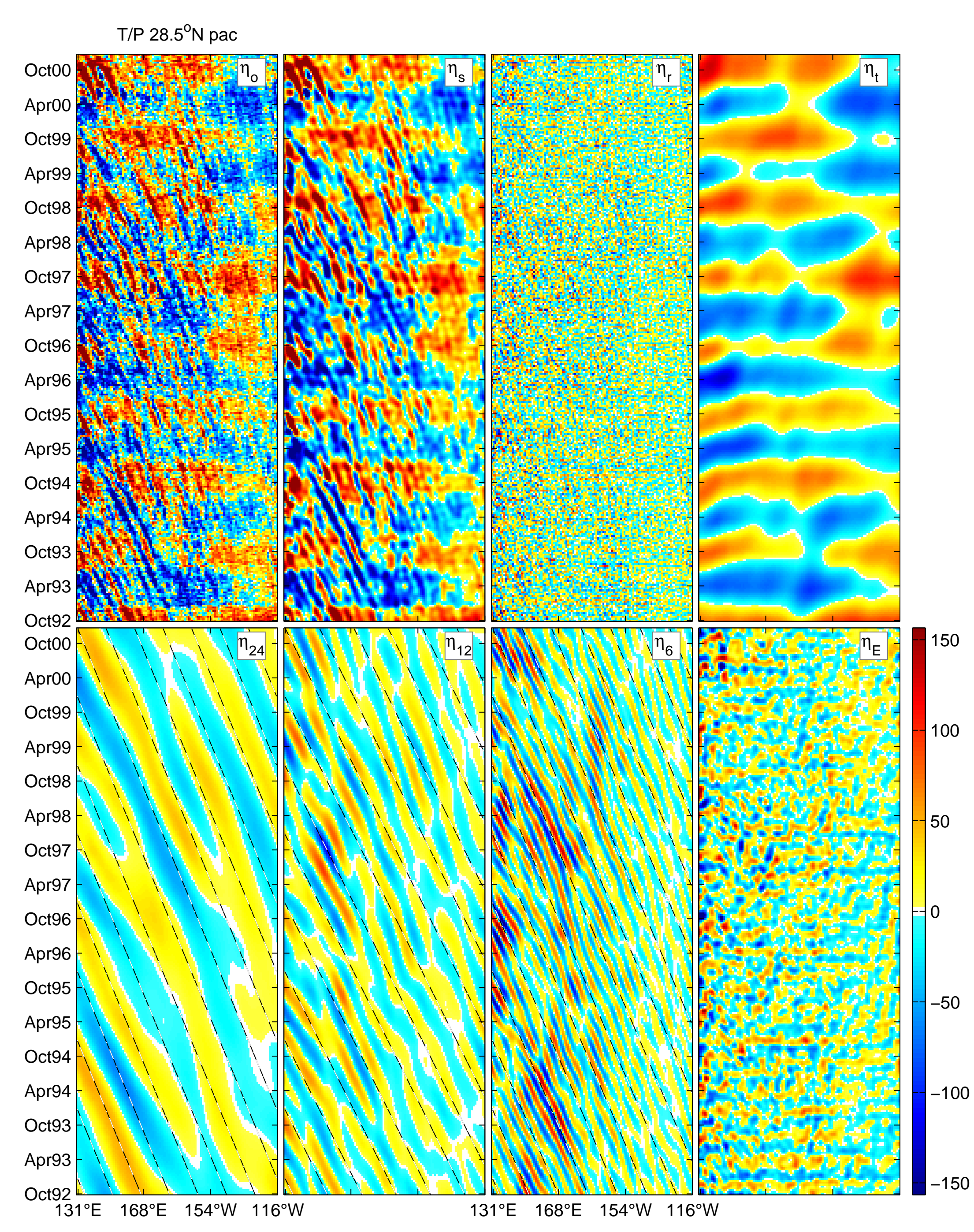

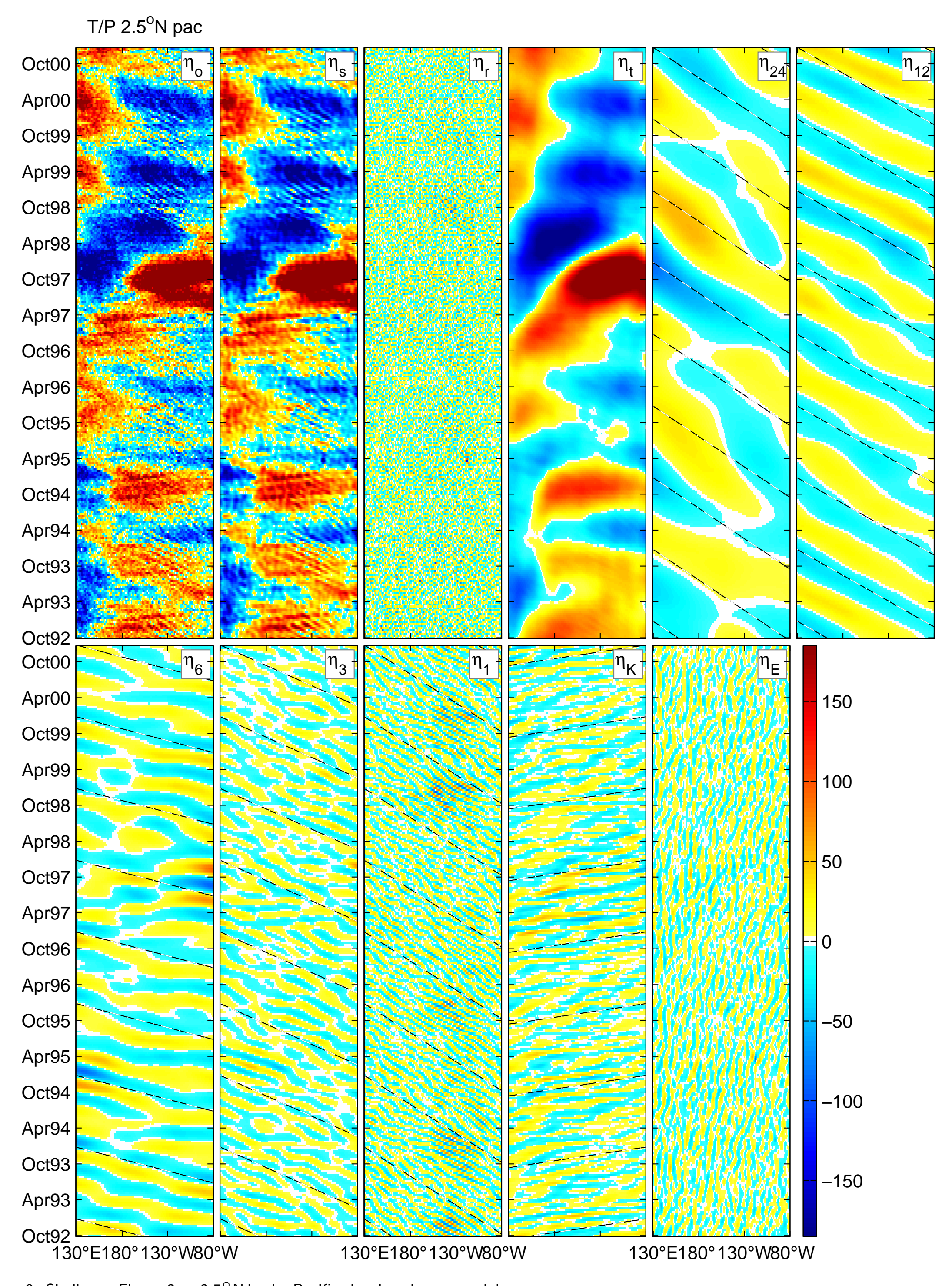

- There is a bias towards high values poleward of $\sim 30^{\circ}$ noticeable in Figures 5, 6 and 7 of $25 \%$, much less than the factor of 2

- The most important difference with respect to $\mathrm{CS}$ is that here the spectral bands are treated separately. It is possible that the remainder of the seasonal signal has biased the $\mathrm{CS} c_{p}$ esti-
mates, which were based on the Radon transform, towards high values.

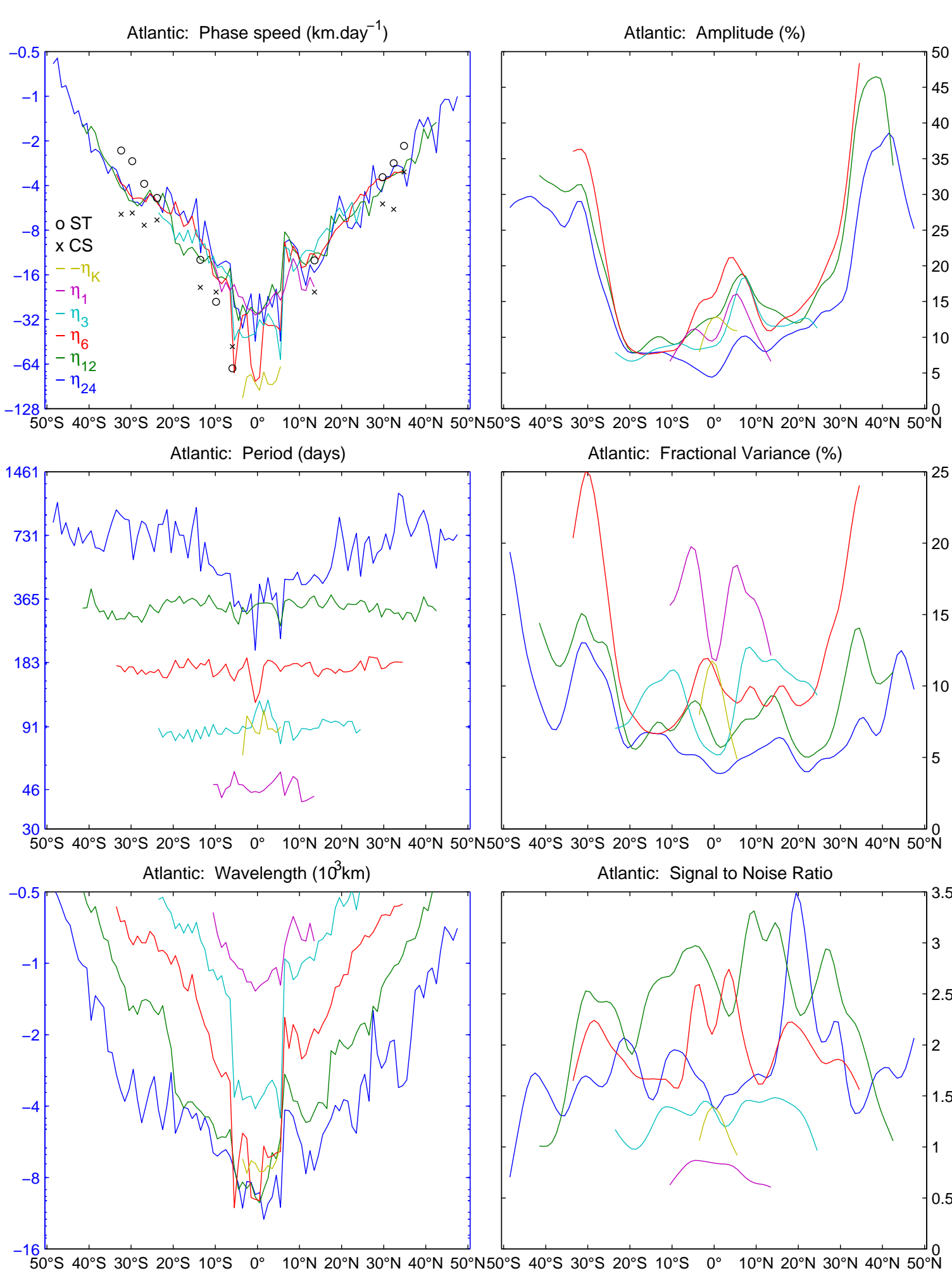

- Outside the equatorial region the $c_{p}$ of the Rossby waves is
indicative of the first baroclinic mode. An implication of this result is that the waves obserced by the altimeter an a this face manifestation of the vertical displacement of the main thermocline.

- These waves change the local amount of heat stored in the water column, which surpasses that of the atmosphere by orimportant influence on the local climate variability.

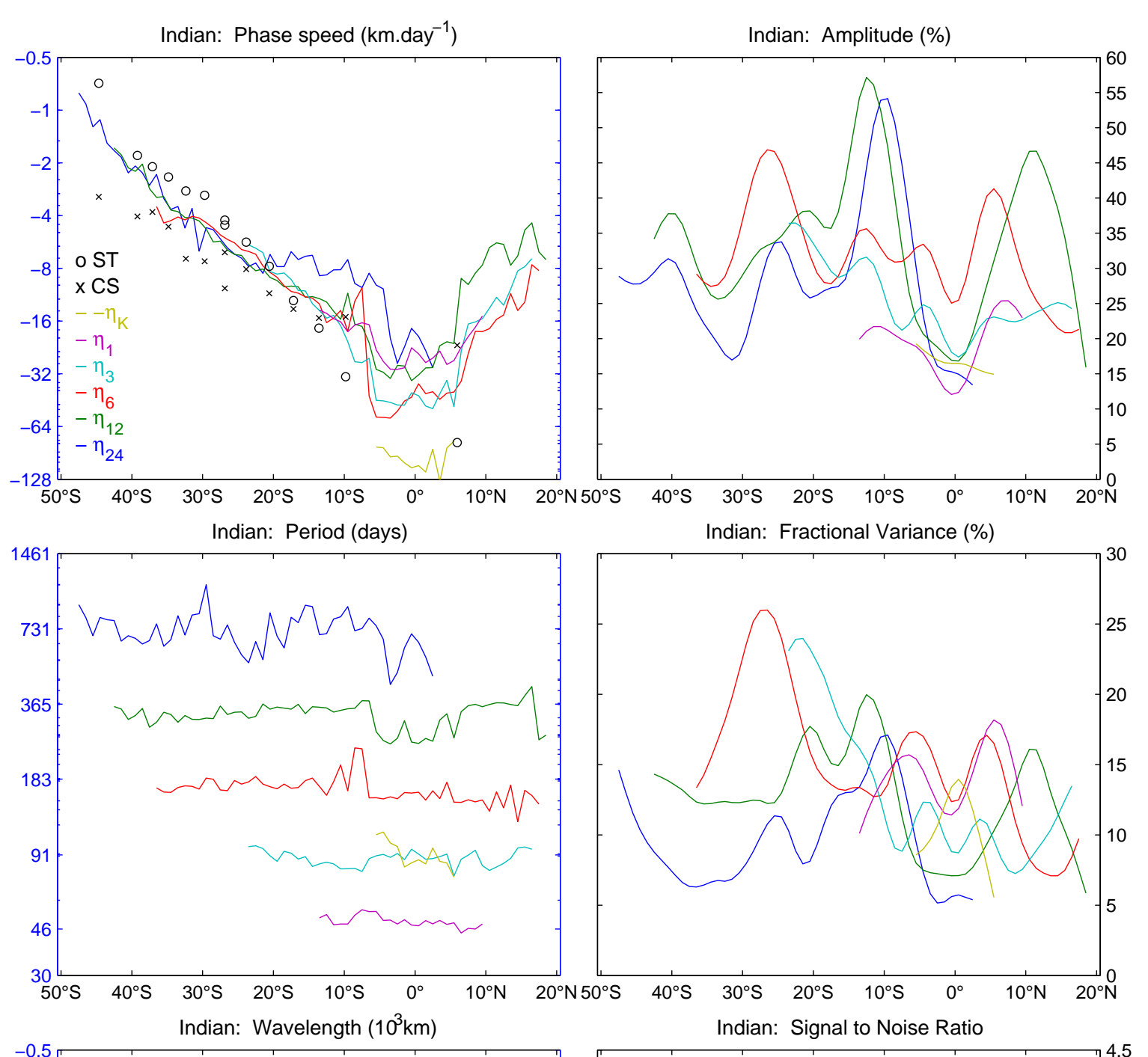

References

[Chelton and Schlax(1996)] Chelton, D. B., and M. Schlax, Global observations of oceanic Rossby waves, Science, 272, 234-238,

[Killworth et al.(1997)] Killworth, P. D., D. B. Chelton, and R. A de Szoeke, The speed of observed and theoretical long extratropi-
cal planetary waves, Journal of Physical Oceanography, 27, 1946cal planetary

[Polito and Cornillon(1997)] Polito, P. S., and P. Cornillon, Long Geophysical Research, $109,3215-3235,1997$.

[Polito et al.(2000)] Polito, P. S., O. T. Sato, and W. T. Liu, Characterization and validation of heat storage variability from ical Research, 105(C7), 16,911-16,921, 2000.

[Sato et al.(1999)] Sato, O. T., P. S. Polito, and W. T. Liu, The importance of in situ salinity for altimeter heat storage estimation Geophysical Research Letters, 27(4), 549-551, 1999.

[Zang and Wunch(1999)] Zang, X., and C. Wunch, The observed dispersion relationship for North Pacific Rossby wave motions, Journal of Physical Oceanography, 29, 2183-2190, 1999.

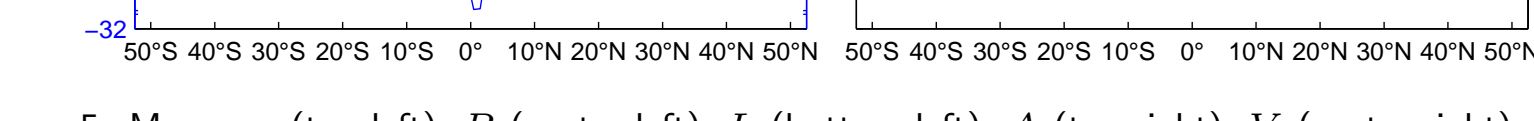

Figure 6: Similar to figure 5 for the Atantic ccean

$\begin{array}{lll}\text { For more details, please e-mail us: } & \\ \text { Paulo S. Polito } & \text { (polito@ltid.inpe.br) }\end{array}$ W. Timothy Liu (liu@pacific.jpl.nasa.gov). 